\title{
SCIENTIFIC REPORTS

\section{OPEN Association between Plasma N-6 Polyunsaturated Fatty Acids Levels and the Risk of Cardiovascular Disease in a Community-based Cohort Study}

\author{
Wei-Sin Yang ${ }^{1,6}$, Yun-Yu Chen ${ }^{1,2,6}$, Pei-Chun Chen ${ }^{3}$, Hsiu-Ching Hsu ${ }^{4}$, Ta-Chen Su ${ }^{4}$, \\ Hung-Ju Lin ${ }^{4}$, Ming-Fong Chen ${ }^{4,5}$, Yuan-Teh Lee ${ }^{4}$ \& Kuo-Liong Chien ${ }^{1,4^{*}}$
}

\begin{abstract}
Most studies support that saturated fatty acid replacement with polyunsaturated fatty acids (PUFAs) may reduce the risk of cardiovascular diseases (CVDs) and put emphasis on the effects of $\mathrm{N}-3$ PUFAs. The reported relationships between N-6 PUFAs and CVD risks vary. We aimed to examine the associations between N-6 PUFA concentrations and CVD risks. In this community-based prospective cohort study on CVD-free patients at baseline ( $N=1835$, age: $60.6 \pm 10.5$ years, women: $44.5 \%$ ), we measured the fatty acid concentrations in the blood using gas chromatography. Four hundred twentyfour participants developed CVDs during follow up. The total N-6 PUFA concentration was inversely associated with the CVD risk, with a $48 \%$ lower risk in the highest N-6 PUFA concentration quartile (hazard ratio $=0.52 ; P$ for trend $<0.001$ ). The estimated population attributable risk of $\mathrm{N}-6$ PUFAs indicated that approximately $20.7 \%$ of CVD events would have been prevented if the plasma N-6 PUFA concentration had been higher than the median value. The total N-6 PUFA concentration presented the highest net reclassification improvement ( $\mathrm{NRI}=7.2 \%, P=0.03$ ) for predicting incident CVD. Further studies on N-6 PUFAs, diet habits, and their relationships with healthcare are warranted.
\end{abstract}

Atherosclerosis is a dynamic inflammatory process and one of the major underlying causes of cardiovascular diseases (CVDs), which may lead to great economic burden owing to its complications and related medical care ${ }^{1}$. From a clinical perspective, determining how to reduce inflammation-associated atherosclerosis may help target therapeutics beyond medications and improve daily clinical practice. The effects of dietary fat on atherosclerosis may be influenced by the inflow of lipids and lipoproteins, such as low-density lipoprotein (LDL) and high-density lipoprotein (HDL), from the plasma to the arterial wall ${ }^{2}$. Many normal metabolic processes cannot be carried out without a supply of essential fatty acids and metabolic enzymes. If essential fatty acids and metabolic enzymes are lacking, the synthesis of eicosanoids would be compromised ${ }^{3-6}$.

Most studies support the idea that saturated fatty acid (SFA) replacement with polyunsaturated fatty acids (PUFAs) may reduce the CVD risk ${ }^{7,8}$. Further, prior studies have demonstrated the plasma cholesterol-lowering effect of PUFAs in the human diet ${ }^{9-11}$. PUFAs are a family of lipids, including some subgroups (e.g. N-3 PUFAs [first double bond at carbon number 3] and N-6 PUFAs [first double bond at carbon number 6] ${ }^{12}$. For example, alpha-linolenic acid (ALA, C18:3 n-3), eicosapentaenoic acid (EPA, C20:5 n-3), and docosahexaenoic acid (DHA, C22:2 n-3) belong to the N-3 PUFA family. Conversely, linoleic acid (LA, C18:2 n-6), gamma-linolenic acid (GLA, C18:3 n6), and arachidonic acid (AA, C20:4 n-6) belong to the N-6 PUFA family ${ }^{3,5}$. In addition, delta-5

${ }^{1}$ Institute of Epidemiology and Preventive Medicine, College of Public Health, National Taiwan University, Taipei, Taiwan. ${ }^{2}$ Division of Cardiology, Department of Medicine, Taipei Veterans General Hospital, Taipei, Taiwan. ${ }^{3}$ Department of Public Health, China Medical University, Taichung, Taiwan. ${ }^{4}$ Department of Internal Medicine, National Taiwan University Hospital, Taipei, Taiwan. ${ }^{5}$ Cardiovascular Research Laboratory, Cardiovascular Center, Clinical Outcome Research and Training Center, Big Data Center, China Medical University Hospital, China Medical University, Taichung, Taiwan. ${ }^{6}$ These authors contributed equally: Wei-Sin Yang and Yun-Yu Chen. *email: klchien@ ntu.edu.tw 


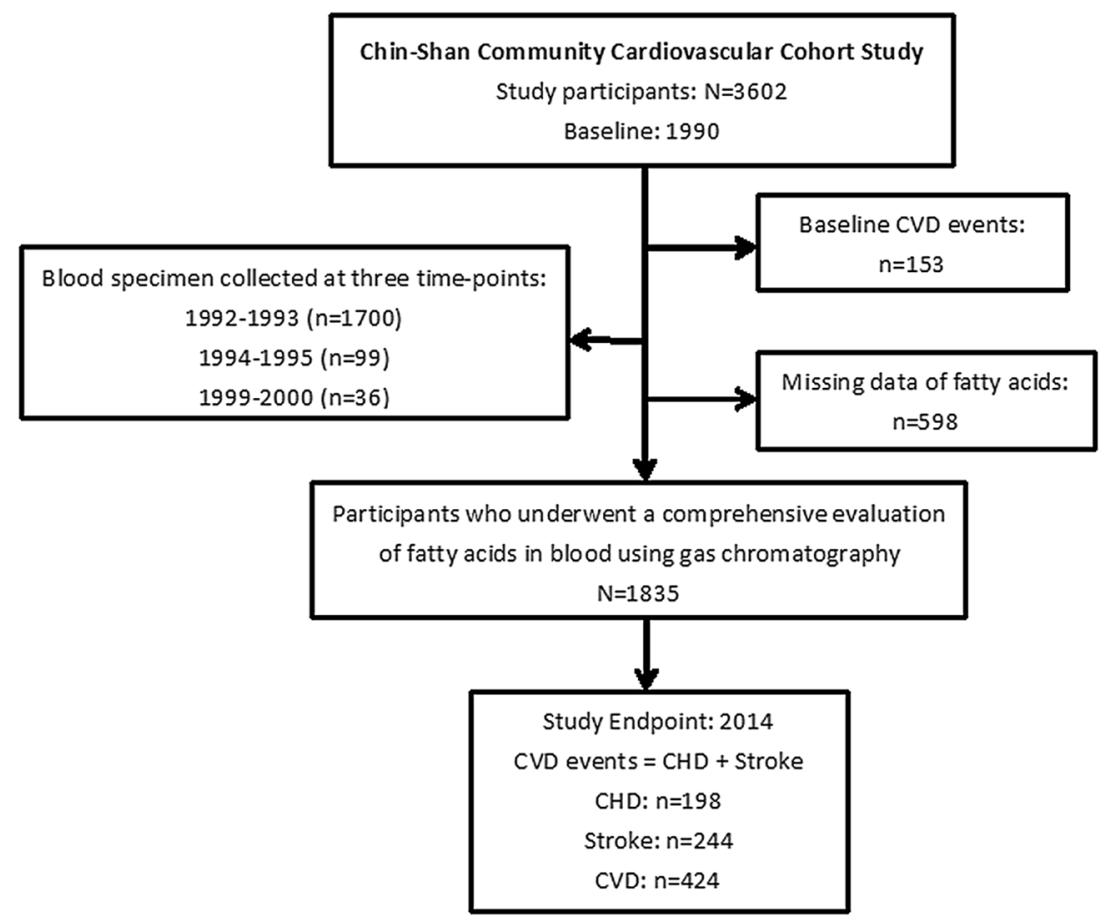

Figure 1. Flow chart of this study. CVD: cardiovascular diseases; CHD: coronary heart diseases.

desaturase (D5D) and delta-6 desaturase (D6D) are the key enzymes involved in the metabolism of both N-3 and N-6 PUFAs, allowing the formation of long-chain metabolites ${ }^{13,14}$. Alterations in D5D and D6D activity may cause inflammation-associated diseases, such as type 2 diabetes mellitus $(\mathrm{DM})^{15}$ and CVD ${ }^{16-20}$.

Prior studies put emphasis on the effects of N-3 PUFA consumption and have shown that N-3 PUFAs were correlated with the incident risk of coronary heart diseases (CHDs $)^{12}$ and that N-6 and N-3 PUFAs have competing roles in the synthesis of eicosanoids ${ }^{21}$. Thus, balance intake of N-6 and N-3 PUFAs is necessary to maintain good health. In 2009, the American Heart Association recommended the consumption of at least 5-10\% of energy from N-6 PUFAs ${ }^{22}$. Till date, the results regarding the relationship between N-6 PUFAs and CVD risks have been inconsistent. Moreover, related data in Asian populations are limited ${ }^{23-29}$. In this study, we aimed to examine the associations between higher concentrations of N-6 PUFAs and CVD risks.

\section{Results}

A total of 1,703 men and 1,899 women living in Chin-Shan Township, Taiwan, were enrolled under regular follow-up, all of whom were free from CVD at baseline (Fig. 1) ${ }^{30}$. Table 1 shows the basic characteristics of the participants according to the LA quartiles at baseline. The participants in the upper quartiles were more likely to be younger and have lower systolic blood pressure, diastolic blood pressure, body mass index, triglyceride concentration, LDL cholesterol concentration, fasting glucose concentration, and higher HDL cholesterol concentration than those in the lowest quartile. In terms of plasma fatty acids, the participants in the upper quartiles had lower concentrations of saturated fat, trans fat, D6D, and higher concentrations of N-6 PUFAs (LA, GLA, and AA), N-3 PUFAs (ALA, EPA, and DHA), and D5D than those in the lowest quartiles. Regarding the categorical variables, the participants in the upper LA quartiles were less likely to smoke and consume alcohol, and a smaller percentage was likely to have a history of hypertension or type $2 \mathrm{DM}$.

A total of 424 individuals experienced CVD events during the follow-up period (a median of 15.9 years). After adjusting for multiple factors, the hazard ratio (HR) for the incident CVD risk in the highest quartile of the total N-6 PUFA concentration was 0.52 as compared with that in the lowest quartile (95\% confidence interval $[C I]=0.38-0.71 ; P$ for trend $<0.001$; Table 2$)$, and that for LA was $0.56(95 \% \mathrm{CI}=0.40-0.77$; $P$ for trend $<0.001$; Table 2). Conversely, N-3 PUFAs showed no significant protective effects in our data (total N-6 PUFAs in the highest quartile: $\mathrm{HR}=0.81,95 \% \mathrm{CI}=0.60-1.09 ; P$ for trend $=0.12$; $\mathrm{EPA}$ in the highest quartile: $\mathrm{HR}=0.98,95 \%$ $\mathrm{CI}=0.74-1.3 ; P$ for trend $=0.87$; DHA in the highest quartile: $\mathrm{HR}=1.04,95 \% \mathrm{CI}=0.77-1.41 ; P$ for trend $=0.64$; see Supplementary Table S2).

Regarding the fatty acid metabolic enzymes, D5D was inversely associated with incident CVD, with a 33\% lower risk in Model 2 (adjusted HR in the highest quartile $=0.67,95 \% \mathrm{CI}=0.50-0.91 ; P$ for trend $=0.02$; Table 3 ) among the participants in the highest quartile compared with those in the lowest quartile. However, the significant protective effect was attenuated after adjustment for additional clinical variables in Model 3. Regarding the $\mathrm{P} / \mathrm{S}$ ratio, the $\mathrm{HR}$ in the highest quartile compared with that in the lowest quartile was $0.58(95 \% \mathrm{CI}=0.42-0.80$; $P$ for trend $<0.001$; see Supplementary Table S3).

For the trend of the CVD risk in the progressively increased N-6 PUFA quartile groups in both sexes, despite the significant interaction between N6-PUFAs and sexes, higher N-6 PUFA concentrations were found to be associated 


\begin{tabular}{|c|c|c|c|c|c|}
\hline \multirow[b]{2}{*}{ LA (\% of total FAs) } & \multirow{2}{*}{\begin{tabular}{|l|} 
Q1 $(n=456)$ \\
Mean SD \\
\end{tabular}} & \multirow{2}{*}{\begin{tabular}{|l|} 
Q2 $(\mathrm{n}=459)$ \\
Mean SD \\
\end{tabular}} & \multirow{2}{*}{$\begin{array}{l}\text { Q3 }(n=460) \\
\text { Mean SD }\end{array}$} & \multirow{2}{*}{\begin{tabular}{|l|} 
Q4 $(n=460)$ \\
Mean SD \\
\end{tabular}} & \multirow[b]{2}{*}{ P-value } \\
\hline & & & & & \\
\hline Age, years & $63.4 \pm 10.1$ & $61.6 \pm 9.9$ & $60.3 \pm 10.8$ & $57.1 \pm 10.4$ & $<0.001$ \\
\hline Systolic BP, mmHg & $132.1 \pm 21.3$ & $131.5 \pm 22.0$ & $129.1 \pm 20.9$ & $125.0 \pm 20.5$ & $<0.001$ \\
\hline Diastolic BP, mmHg & $79.1 \pm 11.3$ & $78.1 \pm 11.4$ & $78.4 \pm 11.4$ & $76.1 \pm 10.2$ & $<0.001$ \\
\hline Body mass index, $\mathrm{kg} / \mathrm{m}^{2}$ & $23.6 \pm 3.6$ & $23.5 \pm 3.5$ & $23.2 \pm 3.1$ & $22.9 \pm 3.0$ & 0.02 \\
\hline Triglyceride, mg/dL & $148.5 \pm 112.3$ & $137.0 \pm 101.3$ & $129.3 \pm 97.7$ & $107.9 \pm 69.5$ & $<0.001$ \\
\hline Total cholesterol, mg/dL & $205.9 \pm 47.3$ & $205.4 \pm 48.5$ & $202.5 \pm 43.3$ & $199.2 \pm 44.4$ & 0.11 \\
\hline HDL cholesterol, mg/dL & $45.8 \pm 12.8$ & $46.2 \pm 12.2$ & $46.9 \pm 13.2$ & $49.8 \pm 13.8$ & $<0.001$ \\
\hline LDL cholesterol, mg/dL & $146.4 \pm 46.4$ & $147.0 \pm 47.1$ & $142.2 \pm 41.9$ & $135.8 \pm 42.6$ & $<0.001$ \\
\hline Fasting glucose, mg/dL & $114.8 \pm 35.4$ & $116.7 \pm 42.9$ & $111.0 \pm 35.8$ & $108.7 \pm 24.9$ & $<0.001$ \\
\hline $\mathrm{CRP}, \mathrm{mg} / \mathrm{dL}$ & $0.3 \pm 0.5$ & $0.3 \pm 0.5$ & $0.2 \pm 0.4$ & $0.3 \pm 0.6$ & 0.47 \\
\hline \multicolumn{6}{|l|}{ Plasma fatty acids component ${ }^{\mathrm{a}}$} \\
\hline Saturated fat, $\%$ total FAs & $55.2 \pm 3.6$ & $52.7 \pm 2.4$ & $50.1 \pm 2.2$ & $45.8 \pm 3.1$ & $<0.001$ \\
\hline Polyunsaturated fat, \% total FAs & $24.3 \pm 3.4$ & $28.0 \pm 2.3$ & $30.6 \pm 2.2$ & $35.4 \pm 3.2$ & $<0.001$ \\
\hline $\mathrm{N}-6 \mathrm{FAs}$ \% total FAs & $21.1 \pm 3.0$ & $24.5 \pm 2.0$ & $27.0 \pm 1.9$ & $31.4 \pm 2.7$ & $<0.001$ \\
\hline LA & $10.4 \pm 1.7$ & $13.7 \pm 0.7$ & $16.7 \pm 1.0$ & $21.5 \pm 2.3$ & $<0.001$ \\
\hline GLA & $0.21 \pm 0.10$ & $0.21 \pm 0.09$ & $0.23 \pm 0.10$ & $0.22 \pm 0.10$ & $<0.001$ \\
\hline $\mathrm{AA}$ & $2.5 \pm 0.7$ & $2.8 \pm 0.8$ & $3.2 \pm 0.9$ & $3.7 \pm 1.1$ & $<0.001$ \\
\hline N-3 FAs, \% total FAs & $1.9 \pm 0.8$ & $2.1 \pm 0.7$ & $2.2 \pm 0.7$ & $2.5 \pm 0.8$ & $<0.001$ \\
\hline ALA & $0.4 \pm 0.1$ & $0.4 \pm 0.1$ & $0.5 \pm 0.2$ & $0.6 \pm 0.2$ & $<0.001$ \\
\hline EPA & $0.3 \pm 0.2$ & $0.4 \pm 0.2$ & $0.4 \pm 0.2$ & $0.4 \pm 0.2$ & $<0.001$ \\
\hline DHA & $1.5 \pm 0.6$ & $1.7 \pm 0.6$ & $1.8 \pm 0.6$ & $2.1 \pm 0.7$ & $<0.001$ \\
\hline Trans fat & $11.3 \pm 3.0$ & $9.4 \pm 1.0$ & $8.4 \pm 1.0$ & $6.9 \pm 1.1$ & $<0.001$ \\
\hline${\mathrm{D} 5 \mathrm{D}^{\mathrm{a}}}^{2}$ & $3.4 \pm 1.0$ & $3.9 \pm 1.0$ & $4.1 \pm 1.2$ & $4.5 \pm 1.2$ & $<0.001$ \\
\hline $\mathrm{D}^{2} \mathrm{D}^{\mathrm{a}}$ & $0.022 \pm 0.03$ & $0.015 \pm 0.01$ & $0.014 \pm 0.01$ & $0.01 \pm 0.01$ & $<0.001$ \\
\hline Gender & & & & & 0.17 \\
\hline Men, n (\%) & $266(57.6)$ & $259(56.8)$ & $260(56.8)$ & $234(51.1)$ & \\
\hline Women, n (\%) & $196(42.4)$ & $197(43.2)$ & $198(43.2)$ & $224(48.9)$ & \\
\hline Current smoker & $231(50.0)$ & $193(42.3)$ & $203(44.3)$ & $172(37.6)$ & $<0.001$ \\
\hline Alcohol intake & $168(36.4)$ & $160(35.1)$ & $139(30.4)$ & $128(28.0)$ & 0.02 \\
\hline Marital status & & & & & 0.03 \\
\hline Unmarried & $23(5.0)$ & $19(4.2)$ & $21(4.6)$ & $11(2.4)$ & \\
\hline Married & $355(77.2)$ & $378(83.3)$ & $376(82.3)$ & $391(85.8)$ & \\
\hline Separated & $82(17.8)$ & $57(12.6)$ & $60(13.1)$ & $54(11.8)$ & \\
\hline Education years & & & & & 0.25 \\
\hline$<=9$ years & $449(97.2)$ & $432(94.7)$ & $435(95.0)$ & 439 (95.9) & \\
\hline$>9$ years & $13(2.8)$ & $24(5.3)$ & $23(5.0)$ & $19(4.2)$ & \\
\hline Job & & & & & $<0.001$ \\
\hline No or housewife & $300(64.9)$ & $257(56.4)$ & $253(55.2)$ & $234(51.1)$ & \\
\hline Manual labor & $131(28.4)$ & $134(29.4)$ & $151(33.0)$ & $180(39.3)$ & \\
\hline Official work & $31(6.7)$ & $65(14.3)$ & $54(11.8)$ & $44(9.6)$ & \\
\hline Frequent exercise, yes & $94(20.4)$ & $86(18.9)$ & $70(15.3)$ & $67(14.6)$ & 0.06 \\
\hline Family history of CAD, yes & $34(7.4)$ & $47(10.3)$ & $35(7.6)$ & $39(8.5)$ & 0.37 \\
\hline Hypertension history, yes & $196(42.4)$ & $189(41.5)$ & $170(37.1)$ & $127(27.9)$ & $<0.001$ \\
\hline Type 2 diabetes mellitus, yes & $87(18.8)$ & $90(19.8)$ & $65(14.3)$ & $55(12.3)$ & $<0.001$ \\
\hline
\end{tabular}

Table 1. Basic characteristics of the participants according to the quartiles of linoleic acid (LA). ${ }^{\text {VValues }}$ represent relative weight \% of fatty acids, except for D5D and D6D. AA: arachidonic acid; ALA: alpha linolenic acid; BP: blood pressure; CAD: coronary artery diseases; CRP:C-reactive protein; D5D: delta-5 desaturase; D6D: delta-6 desaturase; DHA: docosahexaenoic acid; EPA: eicosapentaenoic acid; FAs: fatty acids; GLA: gamma-linolenic acid; HDL: high-density lipoprotein; LA: linoleic acid; LDL: low-density lipoprotein; PUFAs: polyunsaturated fatty acids.

with lower CVD risks in both sex groups (all $P$ for trend $<0.05$, see Supplementary Table S4). The CVD risk in the female participants for Q3 (N-6 PUFA quartile) was significantly lower than that for Q1. However, we did not find a significantly lower CVD risk in the male participants for Q3 than for Q1 (see Supplementary Table S4).

As Table 1 shows an opposite proportion in the distribution of LA between the male and female participants, subgroup analyses for LA and N-6 PUFAs were performed via sex stratification after adjusting for multiple confounders. Higher LA quartiles were associated with lower incident CVD event risks in Model 0 (crude effect; 


\begin{tabular}{|c|c|c|c|c|c|c|c|c|}
\hline N-6 PUFAs & Q1 & Q2 & 95\% CI & Q3 & 95\% CI & Q4 & $95 \%$ CI & P-trend \\
\hline Median & 20.99 & 24.61 & & 27.15 & & 31.22 & & \\
\hline Case (n) & 136 & 112 & & 95 & & 81 & & \\
\hline Person-years & 6466.5 & 6687.1 & & 7453.8 & & 8277.6 & & \\
\hline Rates/1000 py & 21 & $\mid 16.7$ & & 12.7 & & 9.8 & & \\
\hline Model 1 & 1 & 0.83 & $(0.64,1.07)$ & 0.66 & $(0.51,0.87)$ & 0.52 & $(0.39,0.69)$ & $<0.001$ \\
\hline Model 2 & 1 & 0.83 & $(0.64,1.08)$ & 0.68 & $(0.52,0.89)$ & 0.51 & $(0.38,0.68)$ & $<0.001$ \\
\hline Model 3 & 1 & 0.87 & $(0.67,1.13)$ & 0.71 & $(0.54,0.94)$ & 0.52 & $(0.38,0.71)$ & $<0.001$ \\
\hline LA & Q1 & Q2 & 95\% CI & Q3 & $95 \% \mathrm{CI}$ & Q4 & 95\% CI & p-trend \\
\hline Median & 10.83 & 13.77 & & 16.62 & & 20.98 & & \\
\hline Case (n) & 121 & 121 & & 107 & & 75 & & \\
\hline Person-years & \begin{tabular}{|l|}
6509.1 \\
\end{tabular} & 6666.7 & & \begin{tabular}{|l|}
7476.3 \\
\end{tabular} & & 8295.5 & & \\
\hline Rates/1000 py & \begin{tabular}{|l|}
18.6 \\
\end{tabular} & 18.2 & & 14.3 & & 9.0 & & \\
\hline Model 1 & 1 & 1.08 & $(0.83,1.40)$ & 0.86 & $(0.66,1.13)$ & 0.56 & $(0.41,0.77)$ & $<0.001$ \\
\hline Model 2 & 1 & 1.08 & $(0.83,1.40)$ & 0.86 & $(0.66,1.13)$ & 0.57 & $(0.42,0.78)$ & $<0.001$ \\
\hline Model 3 & 1 & 1.05 & $(0.80,1.37)$ & 0.87 & $(0.67,1.15)$ & 0.56 & $(0.40,0.77)$ & $<0.001$ \\
\hline $\mathbf{A A}$ & Q1 & Q2 & $95 \% \mathrm{CI}$ & Q3 & $95 \% \mathrm{CI}$ & Q4 & 95\% CI & p-trend \\
\hline Median & 2.02 & 2.62 & & 3.22 & & 4.21 & & \\
\hline Case (n) & 124 & 118 & & 91 & & 91 & & \\
\hline Person-years & 6607.2 & 7039.1 & & 7471 & & 7830.4 & & \\
\hline Rates/1000 py & \begin{tabular}{|l|}
18.8 \\
\end{tabular} & 16.8 & & 12.2 & & 11.6 & & \\
\hline Model 1 & 1 & 0.91 & $(0.70,1.17)$ & 0.69 & $(0.52,0.91)$ & 0.70 & $(0.52,0.92)$ & 0.004 \\
\hline Model 2 & 1 & 0.94 & $(0.73,1.23)$ & 0.68 & $(0.52,0.91)$ & 0.70 & $(0.53,0.94)$ & 0.005 \\
\hline Model 3 & 1 & 0.95 & $(0.73,1.24)$ & 0.72 & $(0.53,0.96)$ & 0.79 & $(0.58,1.07)$ & 0.06 \\
\hline GLA & Q1 & Q2 & $95 \%$ CI & Q3 & $95 \% \mathrm{CI}$ & Q4 & 95\% CI & p-trend \\
\hline Median & 0.12 & 0.17 & & 0.23 & & 0.32 & & \\
\hline Case (n) & 107 & 100 & & 120 & & 97 & & \\
\hline Person-years & 7085.4 & 7198.9 & & 7087.6 & & 7575.7 & & \\
\hline Rates/1000 py & 15.1 & 13.9 & & \begin{tabular}{|l|l}
16.9 \\
\end{tabular} & & 12.8 & & \\
\hline Model 1 & 1 & 0.99 & $(0.75,1.32)$ & 1.23 & $(0.94,1.61)$ & 0.95 & $(0.71,1.26)$ & 0.94 \\
\hline Model 2 & 1 & 0.98 & $(0.74,1.31)$ & 1.17 & $(0.89,1.53)$ & 0.89 & $(0.66,1.19)$ & 0.55 \\
\hline Model 3 & 1 & 0.99 & $(0.74,1.32)$ & 1.19 & $(0.90,1.58)$ & 0.93 & $(0.69,1.25)$ & 0.81 \\
\hline
\end{tabular}

Table 2. Hazard ratio of incidence CVD according to N-6 PUFAs. Model 1: age, gender. Model 2: Model 1+ body mass index, smoking, alcohol consumption habits, marital status, education level, occupation, and regular exercise. Model 3: Model 2+ baseline hypertension, diabetes, continuous high-density lipoprotein and lowdensity lipoprotein cholesterol values. AA: arachidonic acid; CI: confidence interval; CVD: cardiovascular diseases; GLA: gamma-linolenic acid; LA: linoleic acid; PUFAs: polyunsaturated fatty acids; py: person-years.

Table 4) regardless of sex difference. However, we found a significant interaction between LA and $\operatorname{sex}(P=0.003)$ and between the quartiles of LA and sex $(P<0.001)$ in Model 0 . After adjusting for multiple confounders, the female participants achieved more significant benefits in the highest quartile of LA in reducing the incident CVD risk than did the male participants $(\mathrm{HR}=0.57,95 \% \mathrm{CI}=0.35-0.95$; $P$ for trend $=0.005$; Table 4$)$.

LA showed significant protective effects in the two marker analyses in comparison with N-3 PUFAs and D5D (see Supplementary Table S5). The adjusted HRs for the highest LA and N-3 PUFA quartiles were 0.60 (95\% $\mathrm{CI}=0.38-0.93 ; P$ for trend $=0.02)$ and $1.02(95 \% \mathrm{CI}=0.67-1.53 ; P$ for trend $=0.94)$, respectively; the HRs for the highest LA and D5D quartiles were $0.59(95 \% \mathrm{CI}=0.38-0.92 ; P$ for trend $=0.02)$ and $0.98(95 \% \mathrm{CI}=0.64-1.50$; $P$ for trend $=0.91$ ), respectively.

In the receiver operating characteristic curve analysis, the area under the curve increased from 0.64 in the baseline model to 0.65 in the additional N-6 PUFA model (see Fig. 2 for the performance measurement values). The reclassification improvement with and without N-6 PUFAs is listed in Supplementary Table S6. N-6 PUFAs presented the highest NRI for predicting incident CVD than base model (NRI $=7.2 \%, P=0.03$ ), which indicated that only the inclusion of N-6 PUFAs could improve predictions related to CVD (see Supplementary Table S7).

The estimated population attributable risks (PARs) of the N-6 PUFA concentration indicated that approximately $20.7 \%$ of CVD events would have been prevented if the plasma N-6 PUFA concentration had been higher than the median value ( $26 \%$ of the total fatty acids) (see Supplementary Table S8).

\section{Discussion}

We demonstrated several findings in this study: (1) The total N-6 PUFA concentration was inversely associated with the CVD risk when compared across the highest quartile to the lowest quartile. The inverse associations of N-6 PUFAs and LA with the CVD risks were found especially in the female participants; (2) the estimated PAR of N-6 PUFAs indicated that $20.7 \%$ of CVD events would have been prevented if the plasma N-6 PUFA 


\begin{tabular}{|l|l|l|l|l|l|l|l|l|}
\hline D5D & Q1 & Q2 & 95\% CI & Q3 & 95\% CI & Q4 & 95\% CI & P-trend \\
\hline Median & 2.68 & 3.50 & & 4.14 & & 5.37 & & \\
\hline Case (n) & 117 & 110 & & 117 & & 79 & & \\
\hline Person-years & 6706.3 & 7080.1 & & 7214.6 & & 7748.9 & & \\
\hline Rates/1000 py & 17.4 & 15.5 & & 16.2 & & 10.2 & & \\
\hline Model 1 & 1 & 0.85 & $(0.65,1.11)$ & 0.91 & $(0.69,1.18)$ & 0.64 & $(0.48,0.86)$ & 0.01 \\
\hline Model 2 & 1 & 0.88 & $(0.67,1.16)$ & 0.95 & $(0.72,1.25)$ & 0.67 & $(0.50,0.91)$ & 0.02 \\
\hline Model 3 & 1 & 0.90 & $(0.68,1.18)$ & 1.02 & $(0.77,1.35)$ & 0.77 & $(0.56,1.06)$ & 0.16 \\
\hline D6D & Q1 & Q2 & $\mathbf{9 5 \%}$ CI & Q3 & $\mathbf{9 5 \%}$ CI & Q4 & $\mathbf{9 5 \%}$ CI & p-trend \\
\hline Median & 0.007 & 0.011 & & 0.015 & & 0.023 & & \\
\hline Case (n) & 97 & 98 & & 118 & & 110 & & \\
\hline Person-years & 7441.4 & 7312.8 & & 6980.7 & & 7015.1 & & \\
\hline Rates/1000 py & 13 & 13.4 & & 16.9 & & 15.7 & & \\
\hline Model 1 & 1 & 1.06 & $(0.79,1.42)$ & 1.29 & $(0.97,1.71)$ & 1.24 & $(0.93,1.65)$ & 0.09 \\
\hline Model 2 & 1 & 0.97 & $(0.72,1.30)$ & 1.22 & $(0.92,1.63)$ & 1.14 & $(0.85,1.52)$ & 0.21 \\
\hline Model 3 & 1 & 0.93 & $(0.68,1.26)$ & 1.19 & $(0.88,1.59)$ & 1.16 & $(0.86,1.55)$ & 0.15 \\
\hline
\end{tabular}

Table 3. Hazard ratio of incidence CVD according to fatty acids metabolic enzymes D5D and D6D. Model 1: age, gender. Model 2: Model $1+$ body mass index, smoking, alcohol consumption habits, marital status, education level, occupation, and regular exercise. Model 3: Model $2+$ baseline hypertension, diabetes, continuous high-density lipoprotein and low-density lipoprotein cholesterol values. CI: confidence interval; CVD: cardiovascular diseases; D5D: delta-5 desaturase; D6D: delta-6 desaturase; PUFAs: polyunsaturated fatty acids; py: person-years.

\begin{tabular}{|c|c|c|c|c|c|c|}
\hline \multirow[b]{2}{*}{ Gender } & \multirow[b]{2}{*}{ Models } & \multicolumn{4}{|c|}{ Hazard ratio ( $95 \%$ confidence interval) } & \multirow[b]{2}{*}{$P$ trend } \\
\hline & & Q1 & Q2 & Q3 & Q4 & \\
\hline \multirow{5}{*}{ Men } & Median (n) & $10.8(\mathrm{n}=261)$ & $13.7(n=264)$ & $16.6(n=260)$ & $20.8(\mathrm{n}=234)$ & \\
\hline & Model 0 & 1 (reference) & $0.89(0.64,1.25)$ & $0.79(0.56,0.10)$ & $0.57(0.39,0.83)$ & 0.002 \\
\hline & Model 1 & 1 (reference) & $0.96(0.68,1.34)$ & $0.89(0.63,1.25)$ & $0.74(0.51,1.08)$ & 0.11 \\
\hline & Model 2 & 1 (reference) & $0.93(0.66,1.31)$ & $0.86(0.61,1.21)$ & $0.75(0.51,1.11)$ & 0.14 \\
\hline & Model 3 & 1 (reference) & $0.86(0.60,1.22)$ & $0.84(0.59,1.20)$ & $0.71(0.48,1.06)$ & 0.12 \\
\hline \multirow{5}{*}{ Women } & Median (n) & $10.8(\mathrm{n}=195)$ & $13.8(\mathrm{n}=195)$ & $16.6(n=200)$ & $21.1(\mathrm{n}=225)$ & \\
\hline & Model 0 & 1 (reference) & $1.24(0.85,1.82)$ & $0.74(0.48,1.12)$ & $0.42(0.26,0.66)$ & $<0.001$ \\
\hline & Model 1 & 1 (reference) & $1.33(0.91,1.95)$ & $0.82(0.54,1.24)$ & $0.50(0.32,0.81)$ & $<0.001$ \\
\hline & Model 2 & 1 (reference) & $1.37(0.93,2.03)$ & $0.86(0.56,1.31)$ & $0.53(0.33,0.85)$ & 0.001 \\
\hline & Model 3 & 1 (reference) & $1.43(0.96,2.13)$ & $0.92(0.60,1.41)$ & $0.57(0.35,0.95)$ & 0.005 \\
\hline
\end{tabular}

Table 4. Stratified effects of Linoleic acid (LA) by different genders in various models. We tested if variables of gender and LA have statistical interaction effect, and found that a significantly statistical interaction of $\mathrm{LA}^{*}$ gender (interaction $\mathrm{P}=0.003$ ) and quartiles of linoleic acid $(\mathrm{LA}) *$ gender (interaction $\mathrm{P}<0.001$ ) in Model 0. Model 0: crude effect; Model 1: age; Model 2: Model 1+ body mass index, smoking, alcohol consumption habits, marital status, education level, occupation, and regular exercise; Model 3: Model 2+ baseline hypertension, diabetes, continuous high-density lipoprotein and low-density lipoprotein cholesterol values.

concentration had been higher than the median value (26\% of the total fatty acids); (3) we did not find a significant inverse relationship of N-3 PUFAs with incident CVD. In addition, among the metabolic enzymes, D5D reduced the risk of CVD after adjusting for age, body mass index, smoking, alcohol consumption habits, marital status, educational level, occupation, and regular exercise. However, the inverse effect was attenuated after adding additional factors of baseline hypertension, DM, continuous LDL cholesterol concentration, and HDL cholesterol concentration.

Previous studies put great emphasis on N-3 PUFAs, especially on marine PUFAs (EPA + DHA), and have observed a cardio-protective effect ${ }^{31,32}$. However, our study failed to find a significant association between the concentrations of N-3 PUFAs and CVD risk. Another study on marine PUFAs using the same community-based cohort found that the relative risk of CVD events in the highest quartile compared with that in the lowest quartile was 0.88 for EPA $(P$ for trend $=0.54)$ and 1.12 for DHA $(P \text { for trend }=0.94)^{30}$. Despite the longer follow-up period in our study, we still were unable to detect significant protective effects of marine fatty acids. A meta-analysis of 14 randomised trials (involving 20,485 patients with prior CVD) investigated the role of EPA and DHA supplementation in the secondary prevention of CVD. The authors found insufficient evidence for a secondary protective effect of N-3 fatty acid supplements against overall cardiovascular events ${ }^{33}$. 


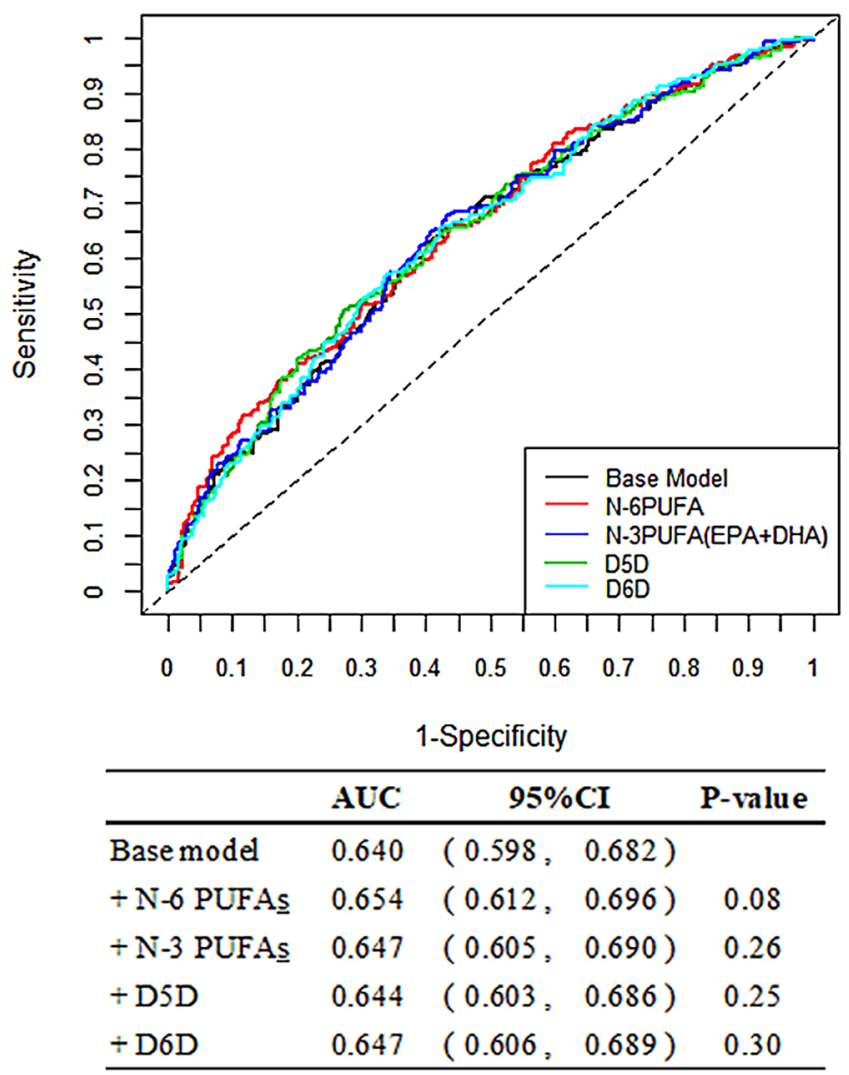

Figure 2. Area under the ROC curves of different fatty acids comparison with the base model. The base model included: age, gender, BMI, smoking, alcohol consumption habits, marital status, education level, occupation, regular exercise, baseline hypertension, diabetes, high-density lipoprotein and low-density lipoprotein cholesterol. AUC: area under the ROC curve; CI: confidence interval; D5D: delta-5 desaturase; D6D: delta-6 desaturase; PUFAs: polyunsaturated fatty acids; ROC: receiver operating characteristic curve.

The clinical value of N-6 PUFAs for total mortality and CVD risks remains controversial ${ }^{21,34}$. In the Framingham Heart Study, higher marine N-3 PUFA concentrations were related to reduced CVD, ischaemic stroke, and mortality risks. However, the authors could not find the same effect in N-6 PUFAs. A meta-analysis pooling 30 prospective cohort studies with nearly 70,000 participants showed that higher concentrations of LA were significantly associated with lower total CVD, cardiovascular mortality, and ischaemic stroke risks ${ }^{35}$, while the concentrations of AA were not associated with cardiovascular outcomes. A systematic review reported that N-3 and N-6 PUFAs have competing roles in the production of anti-inflammatory and inflammatory eicosanoids ${ }^{21}$. Some studies have suggested that the EPA:AA ratio was associated with CHD risk, but they could not find a consistent conclusion regarding the same $\mathrm{e}^{34}$.

Several sources support the proposition that dietary habits may influence plasma fatty acids and health. A prior study analysing 16 cohorts indicated that higher intake of vegetable oils and vegetable foods but lower intake of hard fats and animal foods may reduce the all-cause mortality risk ${ }^{36}$. An ecological study revealed that the 50-year CHD mortality had a significant ecologic correlation with dietary patterns ${ }^{37}$, such as consumption of rich vegetable foods and starch; however, consumption of lower sweet products and animal foods in Mediterranean and Japanese cohorts was inversely correlated with a lower CHD risk. LA could be obtained mainly from vegetable oils (e.g. corn, sunflower, safflower, and soy), accounting for $85-90 \%$ of dietary N-6 PUFAs. In most western countries, emphasis is placed on providing adequate intake of essential nutrients. In clinical practice, although firm requirements have not been established for healthy adults, estimates have been derived from studies in infants and hospitalised patients receiving total parenteral nutrition. These suggested the sufficiency of an LA intake of approximately $0.5-2 \%$ of energy ${ }^{22}$. In this study, we demonstrated that the N-6 PUFA and LA concentrations were inversely associated with the CVD risk, and the significance can be found especially in women and the latter; conversely, the concentrations of N-3 PUFAs were not associated with decreased CVD risks in our cohort during the long-term follow-up period. We failed to explain the actual mechanism behind this finding. However, by reviewing several sources, we believe that various dietary habits, geographic regions, ethnic differences, and changes in oestrogen concentration in women could have potential influences on cardiovascular outcomes. Our research might provide evidence of baseline concentrations of PUFAs on their clinical utility in the relation to the CVD risk, which may be a manifestation indicating the indirect outcome of dietary supplement habits that would allow proper dietary recommendations especially in Asian populations.

The metabolic enzymes, D5D and D6D, have been associated with many chronic diseases and inflammatory responses $^{38,39}$. Hypothetically, a high enzymatic activity may indicate a peculiar susceptibility of the arterial wall 
to inflammatory stimuli during the atherosclerotic process ${ }^{39}$. Higher activities of D6D and lower activities of D5D are associated with the CHD risk ${ }^{29}$. In our study, we also noted a significant inverse dose-response trend of the D5D metabolic enzyme in Model 2. D5D-related processes are a key step in humans, and genetic variations in this step among individuals may regulate the efficiency of the conversion of high concentrations of long-chain PUFAs, such as $\mathrm{AA}^{40}$. Genes suppressing the activity of both D5D and D6D may lead to low plasma and tissue concentrations of PUFAs and their products ${ }^{41}$. The decrease in beneficial eicosanoids could increase inflammatory metabolites that could cause endothelial dysfunction and thus accelerate the progression of low-grade inflammation and atherosclerosis.

The biological mechanisms underlying the relationship of D5D activity and LA with the CVD risk are not well understood. However, the numerous biologically active compounds produced from PUFAs may play a role. In addition to facilitating inflammatory responses, LA and AA metabolites help reduce inflammation and promote resolution ${ }^{25}$. These anti-inflammatory metabolites could inhibit leukocyte activation and platelet aggregation and boost endothelial cell production of other anti-inflammatory metabolites ${ }^{5,42}$. D5D and D6D are the key enzymes that produce long-chain PUFAs, such as AA and EPA. The protective effect of higher D5D activities may be attributed to increased production of anti-inflammatory eicosanoids and lipoxins, resolvins, and protectins from AA, EPA, and DHA ${ }^{39}$. Without the D5D enzyme, dihomo- $\gamma$-linolenic acid cannot be metabolised to long-chain PUFAs that are essential precursors to numerous anti-inflammatory metabolites. Breakdown in the balance between anti- and pro-inflammatory metabolites would then be expected to increase the risk for some chronic diseases.

Our study has several strengths. First, it is a valuable report in Asia with a large sample size and a long-term follow-up period. In addition, the use of a community-based population could help reduce the possibility of selection bias. To control for potential confounding factors, this study also incorporated important socioeconomic and lifestyle factors into several models. We believe a report on the observation of a strong relationship in Asia or Taiwan could provide an important evidence of a potential preventive measure in reducing future CVD risks. Second, we had a clear disease ascertainment strategy. Lastly, we were able to measure fatty acid biomarkers and had good internal standards for measuring plasma fatty acids.

This study also had several potential limitations. First, because we only measured the concentrations of fatty acids once, our results might be attenuated by intra-individual variations. However, Wu et al. also measured plasma fatty acids once and used them as biomarkers from 1992 to $1993^{43}$. Their single measurement still exhibited that the within-person correlations for N-6 PUFAs from baseline to 13 years were moderate (0.49 for LA and 0.60 for AA), showing that the correlation of fatty acids is still high. Furthermore, the actual effect must be stronger because the effects of fatty acids could be underestimated. Second, food frequency questionnaires related to dietary fat intake and dietary supplement habits were not administered. However, we provided adequate data in presenting the associations between N-6 fatty acid concentrations and incident CVD risks, which may be a manifestation indicating the indirect outcome of dietary supplement habits. Lastly, the participants in this study were predominately ethnic Chinese. Our observations might not be generalisable to other ethnic groups.

\section{Conclusion}

In this community-based cohort study in Taiwan, the total N-6 PUFA and LA concentrations were inversely associated with the risk of incident CVD in a dose-response manner. Further clinical trial studies are warranted to investigate the effect of N-6 fatty acid concentrations and dietary habits on cardiovascular health.

\section{Methods}

Study design and population. The design of this cohort study has previously been described ${ }^{44,45}$. Briefly, the study was conducted in Chin-Shan Township, Taiwan, and began in 1990; Interview questionnaires in 2 -year cycles were administered for collecting data on anthropometry, lifestyle, and medical conditions. The study protocol was approved by the Institutional Review Board (IRB number: 201605105RINA) of the National Taiwan University Hospital. Formal informed consents were obtained for all participants for entering the study. Participants were aged over 35 years. All methods were performed in accordance with the relevant guidelines and regulations.

Determination of fatty acid profiles using gas chromatography. Biochemical measurements (including fatty acid profiles) were performed once at baseline in 1835 participants. The procedures used for blood sample collection and fatty acid measurement have been previously reported ${ }^{30,44}$. Briefly, a total of 29 individual fatty acids were identified in this study. The relative quantity of each fatty acid (\% of the total fatty acids) was determined by integrating the area beneath the peak and dividing the results by the total area of all fatty acids $^{30}$. The fatty acid profiles included SFAs, trans fat, polyunsaturated fat, monounsaturated fat, DHA, EPA, and the activity of metabolic enzymes (D5D and D6D). The P/S ratio was defined as PUFAs: SFAs. The inter-assay coefficients of variation were $6.5 \%, 4.3 \%, 4.0 \%$, and $7.5 \%$ for LA, AA, EPA, and DHA, respectively.

Ascertainment of outcomes. The primary endpoints were the time to the first incident CVD events, including CHDs and stroke. Data on incident non-fatal ischaemic and stroke events were obtained from annually administered questionnaires. All diagnoses were confirmed by neurologists and internists. Incident CHD included cases of nonfatal myocardial infarction, angina, and hospitalisation due to percutaneous coronary intervention or coronary bypass surgery. Stroke events included cases of an incident neurological deficit of vascular origin lasting longer than 24 hours and those diagnosed via imaging studies in support of the evidence. The follow-up period ended when the subjects developed new-onset CVD before December 31, 2014, died before December 31, 2014, or lived beyond December 31, 2014. 
Statistical analysis. All participants were categorised into quartiles according to the blood concentrations of the fatty acid profiles and metabolic enzymes. Continuous variables were presented as means \pm standard deviations. The difference among the quartiles was assessed using analysis of variance. The chi-square test was used to test the significance among the categorical data.

To compare the different fatty acids in predicting the CVD risk, we used the following strategies: Multivariable Cox proportional hazard models were used to estimate the HR and their respective 95\% CI (see Supplementary Materials for the details of Models 1-3). All analyses were performed using SAS version 9.3 (SAS Institute, Cary, NC) and R version 3.3.0. Two-tailed $P$-values of $<0.05$ were considered to indicate statistical significance.

Received: 5 October 2018; Accepted: 2 December 2019;

Published online: 17 December 2019

\section{References}

1. Libby, P., Ridker, P. M. \& Maseri, A. Inflammation and Atherosclerosis. Circulation 105, 1135-1143, https://doi.org/10.1161/ hc0902.104353 (2002).

2. Davignon, J. The lipid hypothesis. Arch Surg 113, 28-34 (1978).

3. Calder, P. C. Polyunsaturated Fatty Acids, Inflammation, and Immunity. Lipids 36, 1007-1024 (2001).

4. Tapiero, H., Ba, G. N., Couvreur, P. \& Tew, K. D. Polyunsaturated fatty acids (PUFA) and eicosanoids in human health and pathologies. Biomedicine \& Pharmacotherapy 56, 215-222 (2002).

5. Calder, P. C. Polyunsaturated fatty acids and inflammation. Prostaglandins, Leukotrienes and Essential Fatty Acids 75, 197-202, https://doi.org/10.1016/j.plefa.2006.05.012 (2006).

6. Das, U. N. A defect in the activity of D6 and D5 desaturases may be a factor in the initiation and progression of atherosclerosis. Prostaglandins, Leukotrienes and Essential Fatty Acids 76, 251-268, https://doi.org/10.1016/j.plefa.2007.03.001 (2007).

7. Hu, F. B. et al. Dietary fat intake and the risk of coronary heart disease in women. The New England Journal of Medicine 337, 1491-1499 (1997).

8. Jakobsen, M. U. et al. Major types of dietary fat and risk of coronary heart disease: a pooled analysis of 11 cohort studies. American Journal of Clinical Nutrition 89, 1425-1432, https://doi.org/10.3945/ajcn.2008.27124 (2009).

9. Jackson, R. L., Taunton, O. D., Morrisett, J. D. \& Gotto, A. M. The Role of Dietary Polyunsaturated Fat in Lowering Blood Cholesterol in Man. Circulation Research 42 (1978).

10. Temme, E. H. M., Mensink, R. P. \& Hornstra, G. Comparison of the effects of diets enriched in lauric, palmitic, or oleic acids on serum lipids and lipoproteins in healthy women and men. American Society for Clinical Nutrition 63, 897-903 (1996).

11. Kris-Etherton, P. M. \& Yu, S. Individual fatty acid effects on plasma lipids and lipoproteins: human studies. American Journal of Clinical Nutrition 65, 1628S-1644S (1997).

12. Zuliani, G. et al. The role of polyunsaturated fatty acids (PUFA) in the treatment of dyslipidemias. Curr Pharm Des 15, 4087-4093, https://doi.org/10.2174/138161209789909773 (2009).

13. Petersson, H., Basu, S., Cederholm, T. \& Riserus, U. Serum fatty acid composition and indices of stearoyl-CoA desaturase activity are associated with systemic inflammation: longitudinal analyses in middle-aged men. British Journal of Nutrition 99, 1186-1189, https://doi.org/10.1017/S0007114507871674 (2008).

14. Jacobs, S. et al. Evaluation of various biomarkers as potential mediators of the association between Delta5 desaturase, Delta6 desaturase, and stearoyl-CoA desaturase activity and incident type 2 diabetes in the European Prospective Investigation into Cancer and Nutrition-Potsdam Study. The American Journal of Clinical Nutrition 102, 155-164, https://doi.org/10.3945/ajcn.114.102707 (2015).

15. Kroger, J. \& Schulze, M. B. Recent insights into the relation of Delta5 desaturase and Delta6 desaturase activity to the development of type 2 diabetes. Current Opinion in Lipidology 23, 4-10, https://doi.org/10.1097/MOL.0b013e32834d2dc5 (2012).

16. Warensjö, E., Sundström, J., Vessby, B., Cederholm, T. \& Risérus, U. Markers of dietary fat quality and fatty acid desaturation as predictors of total and cardiovascular mortality: a population-based prospective study. The American Journal of Clinical Nutrition 88, 203-209 (2008).

17. Martinelli, N. et al. FADS genotypes and desaturase activity estimated by the ratio of arachidonic acid to linoleic acid are associated with inflammation and coronary artery disease. The American Journal of Clinical Nutrition 88, 941-949 (2008).

18. Lu, Y. et al. Dietary n-3 and n-6 polyunsaturated fatty acid intake interacts with FADS1 genetic variation to affect total and HDLcholesterol concentrations in the Doetinchem Cohort Study. The American Journal of Clinical Nutrition 92, 258-265, https://doi. org/10.3945/ajen.2009.29130 (2010).

19. Do, H. J., Chung, H. K., Moon, J. \& Shin, M. J. Relationship between the estimates of desaturase activities and cardiometabolic phenotypes in Koreans. Journal of Clinical Biochemistry and Nutrition 49, 131-135, https://doi.org/10.3164/jcbn.10/147 (2011).

20. Kim, S. R., Jeon, S. Y. \& Lee, S.-M. The association of cardiovascular risk factors with saturated fatty acids and fatty acid desaturase indices in erythrocyte in middle-aged Korean adults. Lipids in Health and Disease 14, 133, https://doi.org/10.1186/s12944-0150135-x (2015).

21. Saini, R. K. \& Keum, Y. S. Omega-3 and omega-6 polyunsaturated fatty acids: Dietary sources, metabolism, and significance - A review. Life Sci 203, 255-267, https://doi.org/10.1016/j.lfs.2018.04.049 (2018).

22. Harris, W. S. et al. Omega-6 fatty acids and risk for cardiovascular disease: a science advisory from the American Heart Association Nutrition Subcommittee of the Council on Nutrition, Physical Activity, and Metabolism; Council on Cardiovascular Nursing; and Council on Epidemiology and Prevention. Circulation 119, 902-907, https://doi.org/10.1161/CIRCULATIONAHA.108.191627 (2009).

23. Mozaffarian, D. et al. Interplay Between Different Polyunsaturated Fatty Acids and Risk of Coronary Heart Disease in Men. Circulation 111, 157-164 (2005).

24. Laaksonen, D. E., Nyyssönen, K., Niskanen, L., Rissanen, T. H. \& Jukka T. S. Prediction of Cardiovascular Mortality in Middle-aged Men by Dietary and Serum Linoleic and Polyunsaturated Fatty Acids. Archives of internal medicine 165 (2005).

25. Fritsche, K. L. Too much linoleic acid promotes inflammation-doesn't it? Prostaglandins, Leukotrienes \& Essential Fatty Acids (PLEFA) 79, 173-175, https://doi.org/10.1016/j.plefa.2008.09.019 (2008).

26. Czernichow, S., Thomas, D. \& Bruckert, E. n-6 Fatty acids and cardiovascular health: a review of the evidence for dietary intake recommendations. British Journal of Nutrition 104, 788-796, https://doi.org/10.1017/S0007114510002096 (2010).

27. Farvid, M. S. et al. Dietary linoleic acid and risk of coronary heart disease: a systematic review and meta-analysis of prospective cohort studies. Circulation 130, 1568-1578, https://doi.org/10.1161/CIRCULATIONAHA.114.010236 (2014).

28. Harris, W. S. \& Shearer, G. C. Omega-6 fatty acids and cardiovascular disease: friend, not foe? Circulation 130, 1562-1564, https:// doi.org/10.1161/CIRCULATIONAHA.114.012534 (2014).

29. Matthan N. R. et al. Plasma phospholipid fatty acid biomarkers of dietary fat quality and endogenous metabolism predict coronary heart disease risk: a nested case-control study within the Women's Health Initiative observational study. Journal of the American Heart Association 3, https://doi.org/10.1161/JAHA.113.000764 (2014). 
30. Chien, K. L. et al. Comparison of predictive performance of various fatty acids for the risk of cardiovascular disease events and allcause deaths in a community-based cohort. Atherosclerosis 230, 140-147, https://doi.org/10.1016/j.atherosclerosis.2013.06.015 (2013).

31. Mozaffarian, D. \& Wu, J. H. Omega-3 Fatty Acids and Cardiovascular Disease Effects on Risk Factors, Molecular Pathways, and Clinical Events. Journal of the American College of Cardiology 58, 2047-2067, https://doi.org/10.1016/j.jacc.2011.06.063 (2011).

32. Bucher, H. C., Hengstler, P., Schindler, C. \& Meier, G. N-3 Polyunsaturated Fatty Acids in Coronary Heart Disease: A Meta-analysis of Randomized Controlled Trials. The American Journal of Medicine 112, 298-304 (2002).

33. Kwak, S. M., Myung, S. K., Lee, Y. J. \& Seo, H. G., Korean Meta-analysis Study, G. Efficacy of omega-3 fatty acid supplements (eicosapentaenoic acid and docosahexaenoic acid) in the secondary prevention of cardiovascular disease: a meta-analysis of randomized, double-blind, placebo-controlled trials. Archives of internal medicine 172, 686-694, https://doi.org/10.1001/ archinternmed.2012.262 (2012).

34. Harris, W. S., Tintle, N. L. \& Ramachandran, V. S. Erythrocyte n-6 Fatty Acids and Risk for Cardiovascular Outcomes and Total Mortality in the Framingham Heart Study. Nutrients 10, https://doi.org/10.3390/nu10122012 (2018).

35. Marklund, M. et al. Biomarkers of Dietary Omega-6 Fatty Acids and Incident Cardiovascular Disease and Mortality. Circulation 139, 2422-2436, https://doi.org/10.1161/circulationaha.118.038908 (2019).

36. Menotti, A., Kromhout, D. \& Puddu, P. E. Baseline fatty acids, food groups, a diet score and 50-year all-cause mortality rates. An ecological analysis of the Seven Countries Study. Ann Med 49, 718-727, https://doi.org/10.1080/07853890.2017.1372622 (2017).

37. Kromhout, D., Menotti, A., Alberti-Fidanza, A. \& Puddu, P. E. Comparative ecologic relationships of saturated fat, sucrose, food groups, and a Mediterranean food pattern score to 50-year coronary heart disease mortality rates among 16 cohorts of the Seven Countries Study. Eur J Clin Nutr 72, 1103-1110, https://doi.org/10.1038/s41430-018-0183-1 (2018).

38. Nakamura, M. T. \& Nara, T. Y. Structure, function, and dietary regulation of delta6, delta5, and delta9 desaturases. Annual Review of Nutrition 24, 345-376, https://doi.org/10.1146/annurev.nutr.24.121803.063211 (2004).

39. Tosi, F., Sartori, F., Guarini, P., Olivieri O. \& Martinelli N. In Oxidative Stress and Inflammation in Non-communicable Diseases Molecular Mechanisms and Perspectives in Therapeutics Vol. 824 61-81 (Springer International Publishing, 2014).

40. Mathias, R. A. et al. FADS genetic variants and omega-6 polyunsaturated fatty acid metabolism in a homogeneous island population. The Journal of Lipid Research 51, 2766-2774, https://doi.org/10.1194/jlr.M008359 (2010).

41. Das, U. N. A defect in $\Delta 6$ and $\Delta 5$ desaturases may be a factor in the initiation and progression of insulin resistance, the metabolic syndrome and ischemic heart disease in South Asians. Das Lipids in Health and Disease 9 (2010).

42. Das, U. N. Can essential fatty acids reduce the burden of disease(s)? Lipids in Health and Disease 7, 9, https://doi.org/10.1186/1476511X-7-9 (2008).

43. Wu, J. H. et al. Circulating omega- 6 polyunsaturated fatty acids and total and cause-specific mortality: the Cardiovascular Health Study. Circulation 130, 1245-1253, https://doi.org/10.1161/CIRCULATIONAHA.114.011590 (2014).

44. Lee, Y. T. et al. Chin-Shan Community Cardiovascular Cohort in Taiwan-baseline data and five-year follow-up morbidity and mortality. Journal of Clinical Epidemiology 53, 838-846 (2000).

45. Chien, K. L. et al. Plasma Uric Acid and the Risk of Type 2 Diabetes in a Chinese Community. Clin Chem 54, 310-316, https://doi. org/10.1373/clinchem.2007.095190 (2008)

\section{Acknowledgements}

We thank the staff of the Department of Internal Medicine, National Taiwan University Hospital, and the participants of the CCCC study for their valuable contributions.

\section{Author contributions}

W.S.Y., H.J.L., M.F.C. and K.L.C. designed the experiments; P.C.C., H.C.H., T.C.S., Y.T.L. and K.L.C. conducted the research; H.C.H., P.C.C., Y.T.L. and K.L.C. provided essential materials; W.S.Y., Y.Y.C. and K.L.C. performed statistical analysis; W.S.Y., Y.Y.C. and K.L.C. wrote the paper; K.L.C. had primary responsibility for the final content. All authors read and approved the final manuscript. The authors declare no conflicts of interest.

\section{Competing interests}

The authors declare no competing interests.

\section{Additional information}

Supplementary information is available for this paper at https://doi.org/10.1038/s41598-019-55686-7.

Correspondence and requests for materials should be addressed to K.-L.C.

Reprints and permissions information is available at www.nature.com/reprints.

Publisher's note Springer Nature remains neutral with regard to jurisdictional claims in published maps and institutional affiliations.

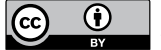

Open Access This article is licensed under a Creative Commons Attribution 4.0 International

License, which permits use, sharing, adaptation, distribution and reproduction in any medium or format, as long as you give appropriate credit to the original author(s) and the source, provide a link to the Creative Commons license, and indicate if changes were made. The images or other third party material in this article are included in the article's Creative Commons license, unless indicated otherwise in a credit line to the material. If material is not included in the article's Creative Commons license and your intended use is not permitted by statutory regulation or exceeds the permitted use, you will need to obtain permission directly from the copyright holder. To view a copy of this license, visit http://creativecommons.org/licenses/by/4.0/.

(c) The Author(s) 2019 